\title{
In vivo evolution of drug-resistant Mycobacterium tuberculosis in patients during long-term treatment
}

\author{
Yuhui $\mathrm{Xu}^{1,2+}$, Fei $\mathrm{Liu}^{3+}$, Suting Chen ${ }^{2,4}$, Jiannan $\mathrm{Wu}^{2,4}$, Yongfei $\mathrm{Hu}^{3}$, Baoli Zhu ${ }^{3^{*}}$ and Zhaogang Sun ${ }^{2,4^{*}}$
}

\begin{abstract}
Background: In the current scenario, the drug-resistant tuberculosis is a significant challenge in the control of tuberculosis worldwide. In order to investigate the in vivo evolution of drug-resistant M. tuberculosis, the present study envisaged sequencing of the draft genomes of 18 serial isolates from four pre-extensively drug-resistant (pre-XDR) tuberculosis patients for continuous genetic alterations.

Results: All of the isolates harbored single nucleotide polymorphisms (SNPs) ranging from 1303 to 1309 with M. tuberculosis H37Rv as the reference. SNPs ranged from 0 to 12 within patients. The evolution rates were higher than the reported SNPs of 0.5 in the four patients. All the isolates exhibited mutations at sites of known drug targets, while some contained mutations in uncertain drug targets including fo/C, proZ, and pyrG. The compensatory substitutions for rescuing these deleterious mutations during evolution were only found in RpoC I491T in one patient. Many loci with microheterogeneity showed transient mutations in different isolates. Ninety three SNPs exhibited significant association with refractory pre-XDR TB isolates.
\end{abstract}

Conclusions: Our results showed evolutionary changes in the serial genetic characteristics of the pre-XDR TB patients due to accumulation of the fixed drug-resistant related mutations, and the transient mutations under continuous antibiotics pressure over several years.

Keywords: Drug-resistant tuberculosis, Treatment, Genetic changes, SNP

\section{Background}

Tuberculosis (TB) is a chronic infectious disease. In recent years, a significant drug resistance, especially multidrug resistance (MDR) and extensive drug resistance (XDR), has occurred [1]. In 2015, there were an estimated 480,000 new cases of MDR-TB and additional 100,000 persons with rifampicin-resistant TB. Moreover, 9.7\% of MDR-TB was found to have XDR-TB, which further increases the difficulty of in treating TB [2]. The effect of genetic mutations in the drug resistance of $M$. tuberculosis has been extensively investigated in a large amount of isolates [3-7]; however, the genetic alterations

\footnotetext{
*Correspondence: zhubaoli@im.ac.cn; sunzg75@hotmail.com

${ }^{\dagger}$ Yuhui Xu and Fei Liu contributed equally to this work.

${ }^{3}$ CAS key Laboratory of Pathogenic Microbiology and Immunology, Institute

of Microbiology, Chinese Academy of Science, Beijing 100101, China

${ }^{2}$ National Tuberculosis Clinical Laboratory, Beijing Chest Hospital, Capital

Medical University, Beijing 101149, China

Full list of author information is available at the end of the article
}

during the course of the long-term treatment, especially at the late stage remain to be deciphered.

The genetic mutations in $M$. tuberculosis after the onset of disease have been analyzed, and the results vary based on the presence or absence of the anti-TB drug treatment. The selection process resulting from the antibiotic therapy may contribute to the occurrence of SNPs. Sun et al. reported that during the treatment, the drug susceptibility of the isolates changed from sensitive strains to resistant strains, and SNPs were acquired to confer the drug resistance [8], which was further confirmed in one XDR case evolved from a susceptible ancestor [9]. However, strains isolated from patients with recurrent tuberculosis exhibited few genetic mutations (0-6 SNPs) between the primary infection and relapse [10]. This was confirmed by the results of a retrospective observational study in which the genetic changes were rarely higher than five SNPs in 3 years and the estimated rate of change in DNA sequences was 0.5 SNPs per

(c) The Author(s). 2018 Open Access This article is distributed under the terms of the Creative Commons Attribution 4.0 International License (http://creativecommons.org/licenses/by/4.0/), which permits unrestricted use, distribution, and 
genome per year $(95 \%$ CI $0.3-0.7)$ in longitudinal isolates [11].

In certain SNPs, the compensatory substitutions are common in drug-resistant $\mathrm{TB}$, which can rescue the deleterious mutations during evolution, and to some extent reflect the transmissibility and prevalence of drug-resistant tuberculosis in a population. With an increase in the number of sequenced isolates, more and more compensatory mutation types have been identified in $M$. tuberculosis [3], especially the compensatory substitutions in RpoA and RpoC for rifampicin-resistant isolates. The compensatory substitutions are also found in the clinical isolates with different genotypes, such as Beijing genotype in northern of China [12], Russia [3, 13] and LAM4/F15/KZN in South Africa [14].

The previous reports have documented the evolution of drug-resistant Mycobacterium tuberculosis in serial $M$. tuberculosis isolates $[8,9,15,16]$. Recently, Andrej et al. reported a view of the heterogeneity of MTBC populations in the host lung [17], but they only focused on the first 8 weeks of treatment and most of them were not MDR. The dynamics of bacterial populations within the host during the course of treatment is not well understood. In this study, deeper characterization of the microbial dynamics within the patient for several years was done based on our results, and a number of $M$. tuberculosis isolates were analyzed over time.

\section{Methods}

\section{Sample selection from patients}

Four patients were recruited from Beijing Chest Hospital. Patients were selected with refractory pulmonary tuberculosis that was confirmed by the clinical symptoms and pathophysiology analysis (based on the positive pathogen cultures and image of the lung by computed tomography). The hematology and blood chemistry, urine biochemistry, mixed bacterial infection, and drug allergic reaction status were observed throughout the routine clinical work.

Eighteen tuberculosis sputum samples from the four refractory tuberculosis patients were collected during treatment through routine clinical work and re-cultured in our laboratory for genome sequencing using Middlebrook $7 \mathrm{H} 9$ medium added with $10 \%$ oleic acid, albumin, dextrose, and catalase (OADC) (BD, MD, USA). Those bacteria were harvested, placed in $1 \mathrm{~mL} 15 \%$ glycerol, and saved in the specimen bank.

\section{Drug susceptibility tests}

The drug susceptibility tests (DSTs) were performed using the absolute concentration method on Lowenstein-Jensen (L-J) slants. The concentration of each of the antibiotics, including isoniazid (INH), rifampin (RIF), streptomycin (SM), pyrazinamide (PZA), ethambutol (EMB), ofloxacin
(OFX), capreomycin (CPM), p-aminosalicylic acid sodium (PAS), ethionamide (ETH), and amikacin (AmK) was indicated in the description [18].

\section{Molecular typing}

The bacterial genotypes were determined using a commercial kit (Isogen Bioscience BV, Maarssen, The Netherlands) according to the reported method by Kamerbeek et al. [19]. The number of tandem repeats at each locus in the isolates was determined based on the number of whole repeats in the PCR product of the size estimated from the gel. PCR for the 12 chosen loci was repeated and compared within and between gels to ensure consistent estimation of the size and tandem repeat copy number [20].

\section{Genome sequencing and assembly}

The genomic DNA of the bacteria was extracted according to the manufacturer's guidelines (Qiagen, Beijing, China) [18]. A 500-bp paired-end library was constructed for each purified DNA sample according to the standard Illumina paired-end protocol with a low-cycle polymerase chain reaction during fragment enrichment. The sequencing was performed on the Illumina Genome Analyzer platform for either 100 or 150 cycles. The low quality reads were filtered using the DynamicTrim and LengthSort Perl scripts in SolexaQA [21]. The short reads were assembled with SOAPdenovo [22] with various length of kmer, a genome assembler developed specifically for next-generation short-read sequences, and the gaps were filled with GapCloser from SOAP after assembling.

The drug-resistant strain M. tuberculosis 11,495 was sequenced using PacBio Single-molecule real-time (SMRT) sequence technology. We used the Hierarchical Genome Assembly Process (HGAP.3) algorithm in SMRT Portal (version 2.2.0) to perform the genome assembly. After sequencing and quality-filtering, 330,992 reads were obtained with a mean length of $3905 \mathrm{bp}$ totaling 1,292,425,796 bp. The complete genome sequence of $M$. tuberculosis 11,495 has a length of 4,428,395 bp and a $\mathrm{G}+\mathrm{C}$ content of $65.6 \%$.

\section{Mutation detection}

M. tuberculosis $\mathrm{H} 37 \mathrm{Rv}$ is the most studied strain of tuberculosis in research laboratories. However, it is becoming apparent that use of $\mathrm{H} 37 \mathrm{Rv}$ as a sole reference genome in analyzing clinical isolates presents some limitations in investigating M. tuberculosis [23]. Hence, we included the genome sequence of a Beijing lineage 11,495 genetically close to our strains in our analyses, in addition to H37Rv. First, the short reads were aligned with two reference genomes using the SOAP2 program [24], which included the standard reference 
strain M. tuberculosis H37Rv (GenBank AL123456), and the other strain 11,495 . Then, SOAPsnp was used to score the SNPs from the aligned reads [25]. The SOAPsnp results were filtered as follows: (1) the read coverage of the SNP site was greater than three, (2) the Illumina quality score of each allele was greater than 30, and (3) the number of mapped best bases was more than two times the number of all mapped second-best bases. SNPs located in PE/PPE and PE-PGRS gene families that might cause incorrect read alignment were also excluded. In addition, SNPs showing sequencing and analysis noise patterns were manually removed. For deletion analysis, we used the results from the aligned reads, and the regions covered by less than three reads were considered as the deletion regions. To confirm SNPs and deletion analysis, we also performed a genome comparison analysis with results from genome assembly by Mauve [26] using default parameters. The correlation coefficient between the number of SNPs and the duration of the treatment was analyzed by the Pearson method. A $p$ value of $<0.05$ was considered statistically significant. Fisher's exact tests [27] were used to assess the statistical significance of the difference in SNPs between our sequenced genomes and the drug-resistant reference Beijing genomes. COG annotation was performed using the BLAST software against the COG database. COG enrichment analysis was determined using Fisher's exact test by comparing the prevalence of a target group of genes assigned to a specific COG category to the prevalence of genes in the whole genome.

\section{Phylogenetic analysis}

Maximum likelihood phylogenetic tree of the $M$. tuberculosis isolates was created using the SNPs of the whole genome sequences by kSNP3 with a k-mer length of 19 [28]. A median joining network can be used to infer intraspecific phylogenies where small genetic mutations are expected. It resolves all possible evolutionary paths connecting the considered taxa and postulates new nodes. Hence, a median-joining tree was created using the Population Analysis with Reticulate Trees (PopArt v1.7) [29].

\section{Results \\ Characteristics of the strains}

Four refractory tuberculosis patients without adjunctive surgery were included in this study. Only one patient was experiencing a first-time hospitalization; the others were re-treatment patients with a long history of tuberculosis before being hospitalized (Table 1). All of the patients exhibited more than $10 \%$ of weight loss during their hospital stay. The abnormal blood chemistry indices included alpha-hydroxybutyric acid and C-reactive protein. In addition, all patients exhibited abnormal hematology indices with higher erythrocyte sedimentation rate and fibrinogen concentration. All patients had normal urine biochemistry.

The implemented treatment regimens followed the WHO guidelines according to the best drug combinations and dosing schedules for multidrug resistance tuberculosis (MDR-TB) and extensive drug resistance tuberculosis (XDR-TB) [30, 31]. However, the patients were still not cured in an adequate amount of time and three of the four cases were resistant to the fourth and fifth group of the anti-TB drugs, such as ETH and PAS (Table 2). Moreover, the drug resistance patterns of the four patients increased, with the progression in treatment. Drug susceptibility tests showed that the initial strains from four patients were pre-XDR before treatment but later became XDR by the end of the study.

\section{Molecular genotype and whole genome sequencing}

PCR-based variable-number tandem repeat (VNTR) analysis was performed using the 12-MIRU-locus method and two patterns were identified, 124127283422 for isolates from patient A and 226126313321 for isolates from patients B, C and D. Both the Spoligotyping (octal codes: 000000000003771) and genome sequencing results showed that all the isolates belonged to the Beijing lineage.

The basic whole-genome sequencing statistics are shown in Additional file 1: Table S1. For each sample, 2.0 to 5.8 million 150 -bp or 100 -bp paired-end reads

Table 1 Patient clinical data

\begin{tabular}{lllllllllllll}
\hline NO. & Diagnosis & DH(y) & Weight loss(kg) & HE & Blood Chemistry & ESR & FC & ID & UB & ARD & OBI & Survival \\
\hline A & IPT & 45 & $9 / 64(14.06 \%)$ & N & LAHTA, HCRP & $11.37 \pm 6.23$ & $4.45 \pm 1.23$ & No & N & RFP, PZA & AHSC, NC & YES \\
B & CFPT & 12 & $8 / 58(13.79 \%)$ & N & NAHBA, HCRP & $21.37 \pm 8.23$ & $6.45 \pm 2.23$ & No & N & NO & AHSC, NC & YES \\
C & IPT & 28 & $17 / 75(22.67 \%)$ & N & LAHBA, HCRP & $35.38 \pm 12$ & $6.06 \pm 1.60$ & No & N & NO & AHSC, NC & YES \\
D & IPT & 0 & $14 / 58(24.13 \%)$ & N & NAHBA, HCRP & $26.67 \pm 2.52$ & $5.64 \pm 1.12$ & No & N & NO & AHSC, NC & YES \\
\hline
\end{tabular}

$D H(y)$ disease history (years), $H E$ hematology, ESR erythrocyte sedimentation rate, $F C$ fibrinogen concentration, $I D$ immunodeficiency, $U B$ urine biochemistry, $A R D$ allergy reaction drugs, $O B I$ other bacterial infection, IPT infiltrative pulmonary tuberculosis, CFPT chronic fibrocavenous pulmonary tuberculosis, $L A H B A$ low alphahydroxybutyric acid, NAHBA normal alpha-hydroxybutyric acid, HCRP high C-reactive protein, AHSC a-hemolytic streptococcus, NC Neisser's coccus, N normal 


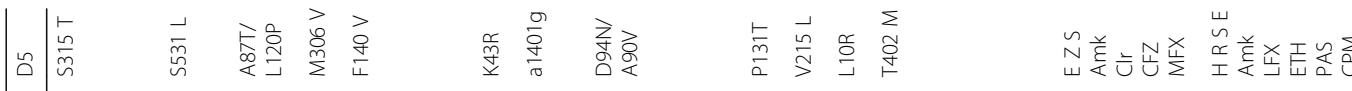

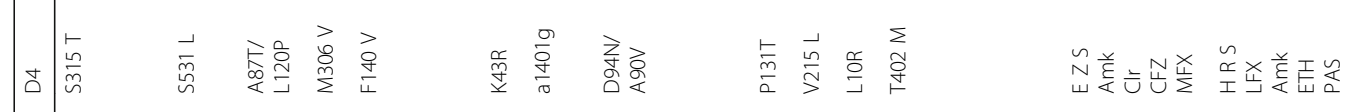

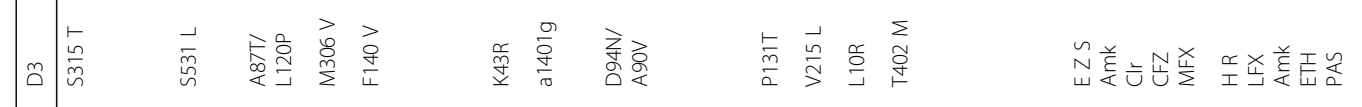

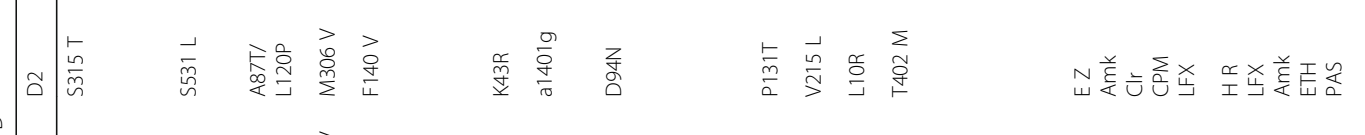

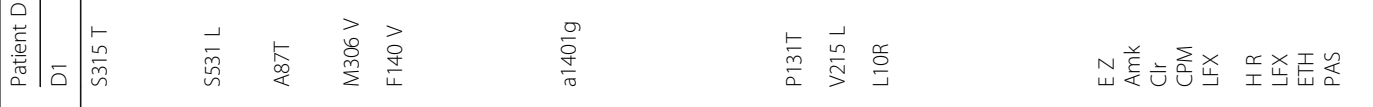

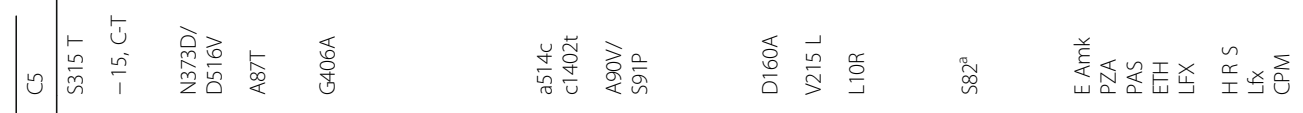

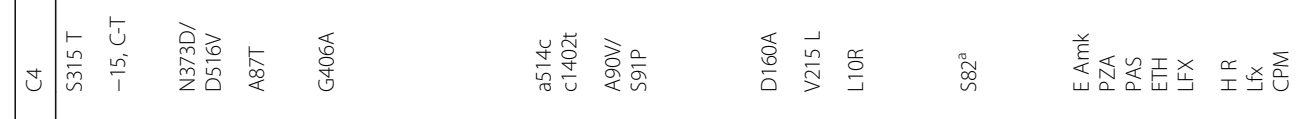

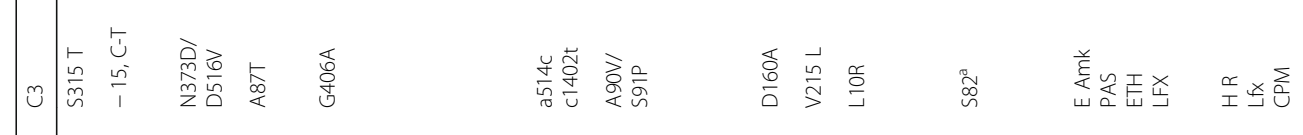

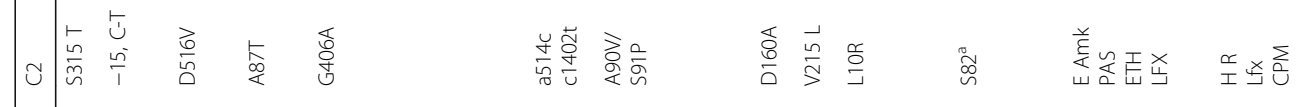

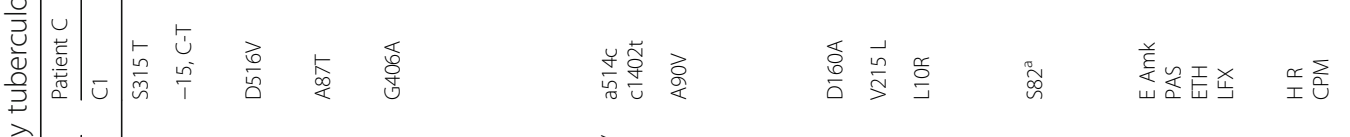

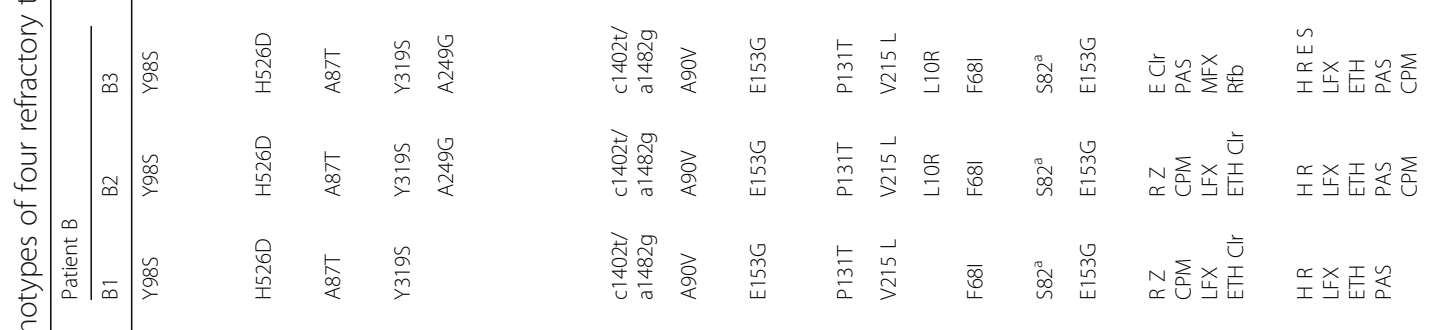

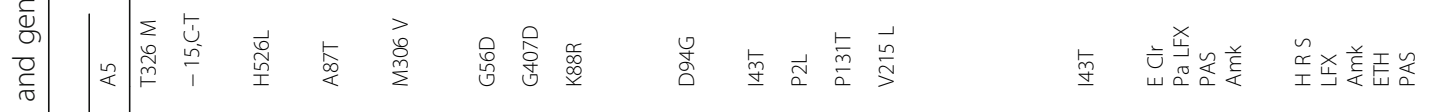

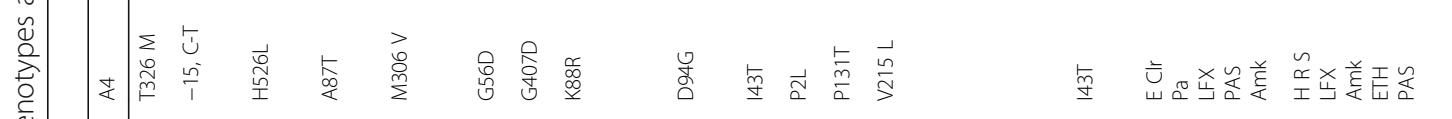

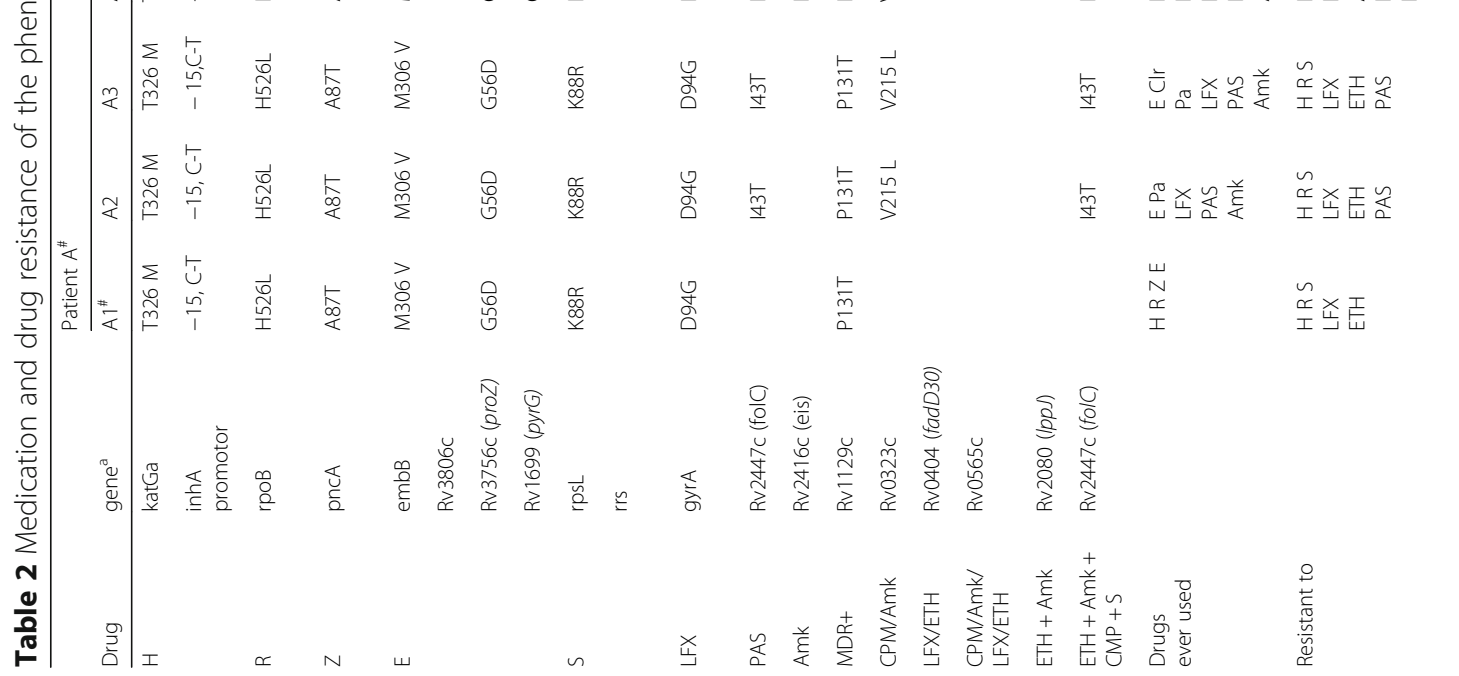


were obtained, which corresponded to an average sequencing depth ranging from 138 to 270 -fold. The GC content of the genomes was approximately $65.5 \%$, as expected for the species. The size of the genomes varied from 4.26 to $4.33 \mathrm{Mb}$.

\section{Phylogenetic analysis of $M$. tuberculosis isolates}

A median-joining tree was created based on the SNPs from draft genome sequences of the 18 clinical $M$. tuberculosis isolates and the complete genomes of four additional M. tuberbulosis strains (Fig. 1). All clinical isolates from the same patients formed separate clades, which indicated that the successive isolates were derived from the same ancestor strain.

\section{SNP and Indel detection}

With M. tuberculosis H37Rv (ATCC 27294) as the reference, the sequencing coverage was approximately $99 \%$, and all of the isolates harbored SNPs ranging from 1303 to 1309 (Fig. 2a, Additional file 1: Table S1). TC, AG, GA, and CT transitions were found to be the most frequent SNP types (Additional file 2: Figure S1), and the mean transversion/transition ratio was 0.60 . With $M$. tuberculosis 11,495 as the reference, the four isolates harbored SNPs ranging from 238 to 276 (Fig. 2a).

Venn diagram showing the SNPs distribution in four isolates under study compared with the H37Rv demonstrates that all the strains used in the experimentation appeared to be quite similar (Fig. 2b): 1157 SNPs were common, while each isolate possessed a few individual SNPs.

The intra-patient isolates exhibited a low SNPs variation ranging from 0 to 12 (Fig. 3a). There were $63.6 \%$ nonsynonymous, $13.6 \%$ synonymous, and $22.7 \%$ noncoding SNPs in intra-patient SNPs; while there were $54.6 \%$ nonsynonymous, $30.4 \%$ synonymous, and $15.0 \%$ noncoding SNPs when compared with H37Rv (Fig. 3b). Mutation rate is the number of SNPs between any two paired isolates per the calendar year, was calculated for each patient. The mean mutation rate was 3.2 SNP per genome per year (Fig. 3c). There was no statistically significant relationship between the treatment time and the SNP number $(p<0.05)$.

When compared to $\mathrm{H} 37 \mathrm{Rv}$, some deleted genes were also identified in more than one of the tested patients (Fig. 4). cut1, plcD, and wag22 genes in all of the isolates were found to be completely deleted.

\section{Mutations related to drug resistance}

In this study, the nonsynonymous mutations were found in many drug target genes (Table 2). The nonsynonymous mutations related to current anti-TB drugs in katG, pncA, gyrA, rpsl, rpoB, embB, ethA, rrs, and $R v 3806 c$ were already confirmed $[5,11]$. The nonsynonymous mutations in katG, rpoB, pncA, $e m b B$, and $g y r A$ were found in all of the 18 isolates,

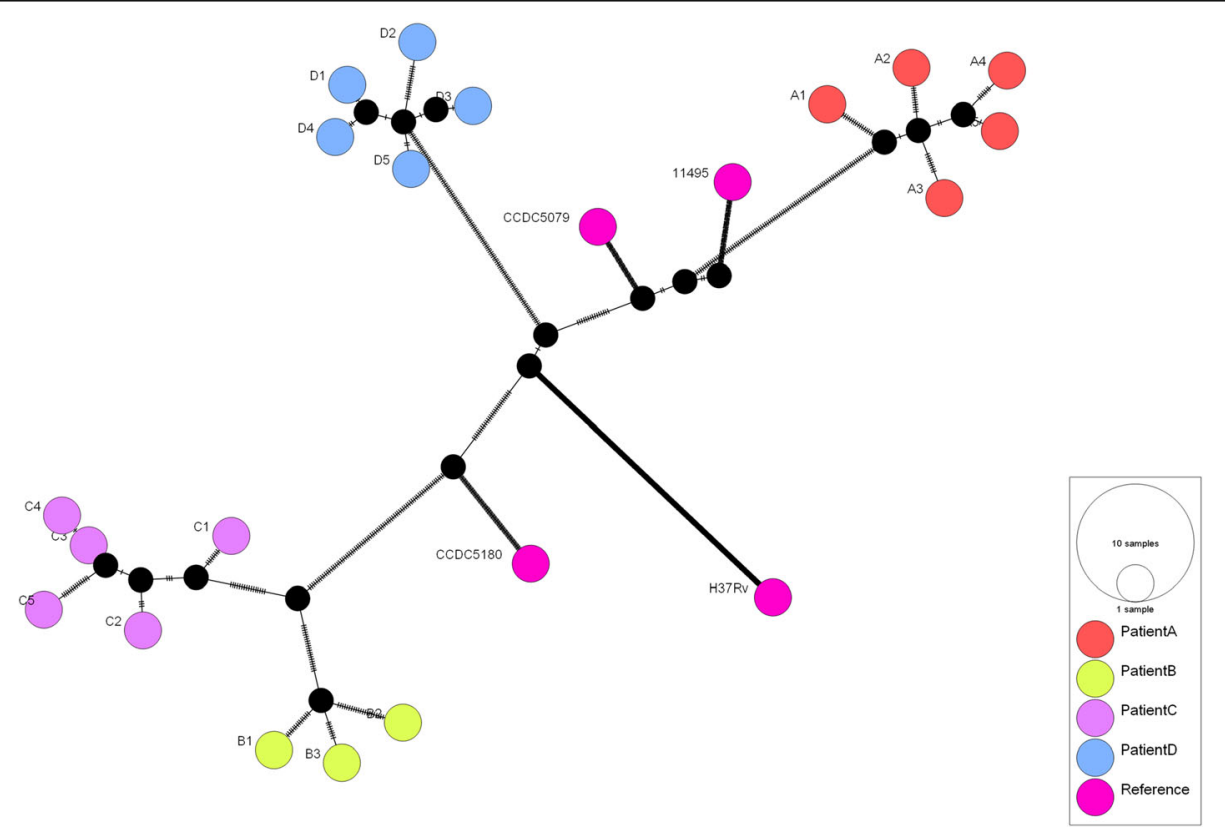

Fig. 1 Median-joining networks based on SNPs in Mycobacterium tuberculosis isolates showing microevolution events. The nodes of the networks correspond to the different MTB isolates. The color inside each circle represents the host. Each short line along the lines, linking the nodes corresponds to a single-nucleotide polymorphism (SNP) detected between the variants in the connected nodes 

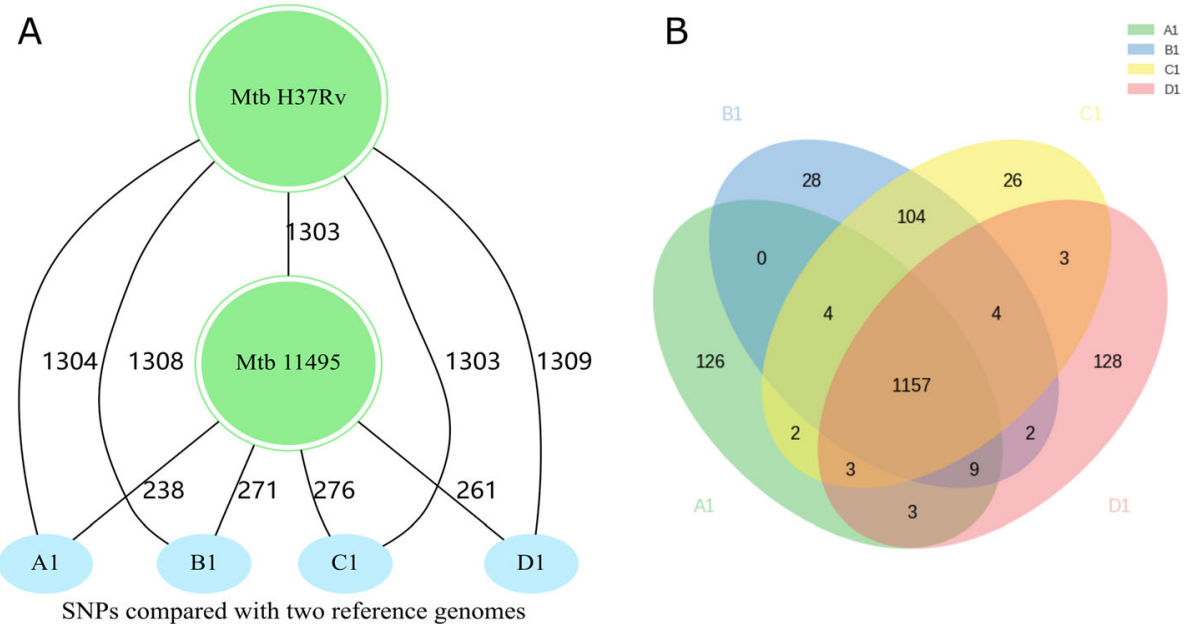

SNPs compared with two reference genomes

Fig. 2 SNPs of the bacterial isolates collected for each patient. a The number of SNPs was assessed relative to H37Rv (NC_000962.3) and 11,495. The number on the line indicates the number of SNPs between the two isolates. $\mathbf{b}$ Venn diagram of different SNPs in each patient relative to reference H37Rv

A

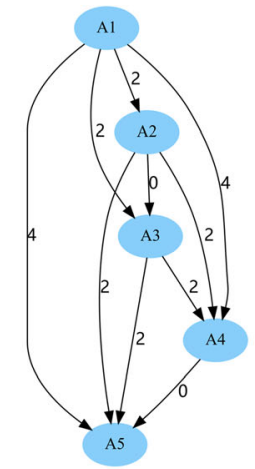

B

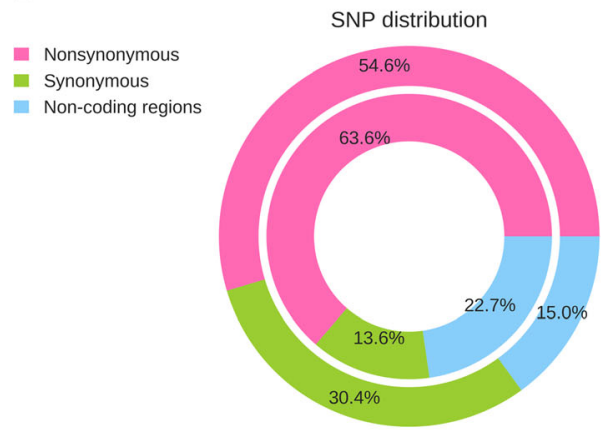

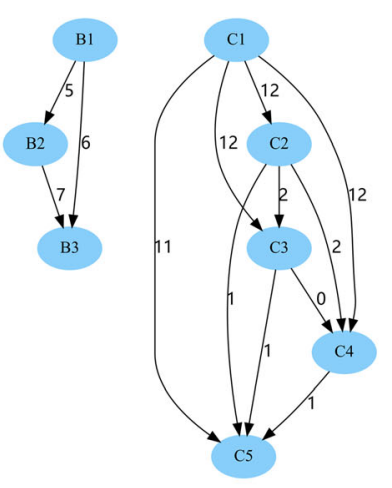

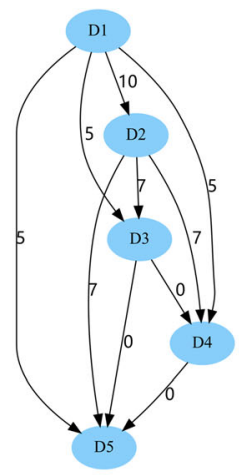

C

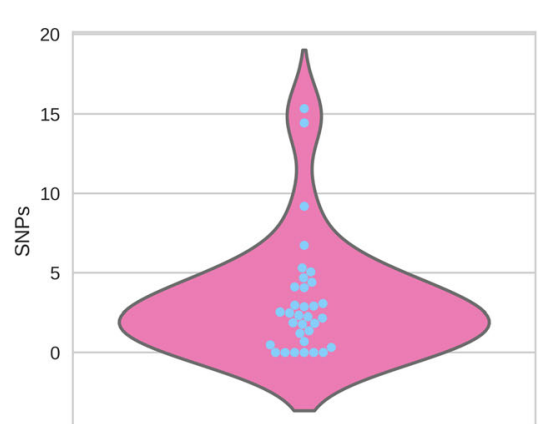

Fig. 3 Intra-patient evolution and mutation rates. a The numbers of SNPs of the bacterial isolates at different stages of treatment for each patient. $A, B, C$, and D represent the four selected patients. The number on the short line indicates the numbers SNPs between two isolates at different time points of treatment of the same patient. b Pie chart depicting distribution of SNPs. Outer: patients compared with H37Rv; Inner: intra-patient. c Violin plot of calculated pairwise mutation rates per year between any pair of strains 


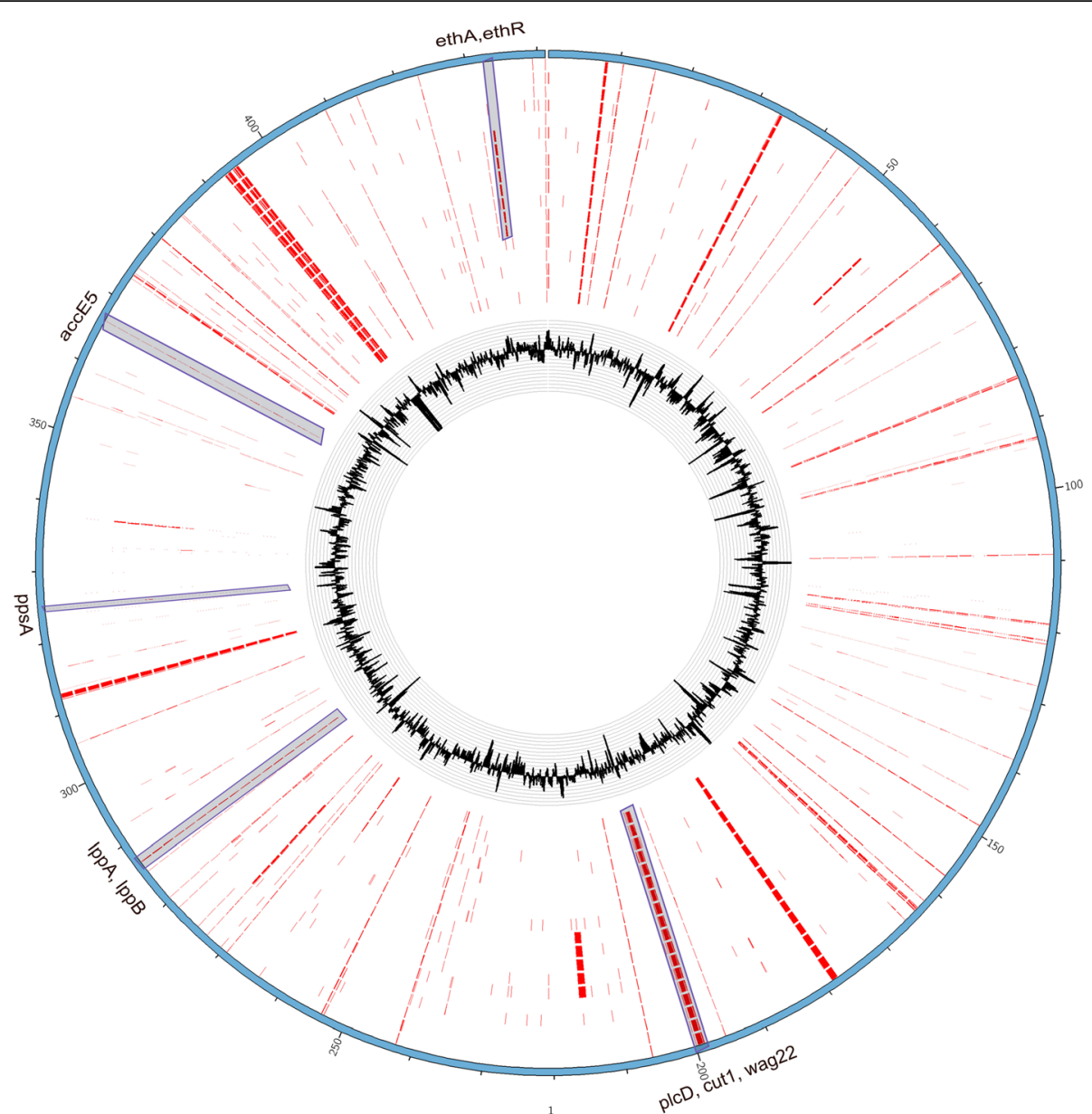

Fig. 4 Comparison of the missed regions among the sequenced genomes. A circular map of the regions (Red color) missed when mapping the raw reads of the sequenced strains against the H37Rv genome (NC_000962.3). Inside track within the map displays a plot of $\mathrm{G}+\mathrm{C}$ contents. In outer strain circles, from the inside out, include the following missed regions in those isolates of $A 1, A 2, A 3, A 4, A 5, B 1, B 2, B 3, C 1, C 2, C 3, C 4, C 5$, D1, D3, D4, and D5. The circular map was constructed using Circos, which is a software package for visualizing data and information (http:// circos.ca/). Genes in the main missed regions were showed (Table 3), not including those belonging to PE or PPE family that contain large, near perfect repeats that create a high likelihood of sequencing and assembly error

and drug-resistance related mutations in rpsl, rrs, ethA, Rv3806c and inhA promoter were found in the isolates from certain patients. No mutation was found in $\operatorname{rps} A$ and $p a n D$ for PZA resistance, in ribD and thy $A$ for PAS resistance, in gidB for SM or Amk resistance, in $e m b A, e m b C$, $e m b R$, iniAC for EMB resistance, and in $a h p C, f a b G 1$ and $i n h A$ for INH resistance (Table 2). Some nonsynonymous mutations that were inferred for drug resistance [32] were also found in part among the isolates, such as $R v 2447 c$ (folC), Rv3756c (proZ), Rv1699 (pyrG), Rv1129c, Rv0323c, Rv0404 (fadD30), Rv0565c, Rv2080 (lppJ) and $R v 3862 c$ (whiB6). We analyzed the occurrence of compensatory substitutions in RpoA and $\mathrm{RpoC}$ in all the sequenced 18 isolates and found that the compensatory substitutions exist only in RpoC at I491T, in isolates from patient $\mathrm{D}$.

\section{Transient mutation}

Apart from the fixed mutations that were related to the drug resistance, several transient mutations was also found in all of the patients (Additional file 3: Table S2). A micro-heterogeneity at some related genome sites of the isolates was found on further examination of the transient mutations based on the raw reads. Interestingly, the same base positions that determined this microheterogeneity were also found in the isolates from different patients.

Next, we investigated all the nonsynonymous SNPs to identify the mutations that could be involved in infection and persistence in all patients. The isolates in patient $A$ harbored a P2L mutation in eis (Rv2416c) which shows to enhance the intracellular survival of $M$. tuberculosis $[33,34]$. In patient $C$, we found a $C 451 Y$ mutation in ponA2 (Rv3682), which is involved in the adaptation of M. tuberculosis to dormancy [35]. 
Confirmation of the SNPs and Indels with published data We downloaded all the raw read sequences reported in China [5] from NCBI's Sequence Read Archive (SRA) by SRA Toolkit and performed de novo genome assembly for all samples by SOAPdenovo [22] with various length of kmer. In order to find the specific SNPs of the tested isolates with Beijing genotypes in China, their draft genome sequences were compared to the reference drug-resistant strains. The phylogenetic trees demonstrated that the tested 18 bacterial isolates are evolutionarily close (Fig. 5). Ninety three SNPs exhibited significantly different percentages (adjusted $p$-value $<$ 0.01 ) between our sequenced genomes and the reference drug-resistant Beijing genomes (Additional file 4: Table S3). Interestingly, we found two nonsense mutations, 1 coding sequence substitution c. 397G > T mutation occurred in $\operatorname{Rv0768~(aldA)~and~one~c.~}$ $245 \mathrm{C}>\mathrm{A}$ mutation occurred in Rv2080 (lppJ).

We further analyzed the distribution of SNPs according to the different categories of the Clusters of Orthologous Groups (COG). Secondary metabolites biosynthesis, transport, and catabolism (class Q), Lipid transport and metabolism (class I), and Energy production and conversion (class C) were the most frequent COG categories (Additional file 5: Figure S2). In addition, we found that they were significantly enriched in Secondary metabolites biosynthesis, transport, and catabolism (class Q; $P$ value $=0.048)$.

In order to confirm the deletions, these results were compared to the reported genome sequences in China [5]. Almost all of the three genes (cut1, plcD, and wag22) were partially deleted in all of the Beijing genotype isolates, and in few of the non-Beijing genotype isolates. There was no difference in the frequency of deletion of genes among the drug-resistant and drug-sensitive isolates. The deletions appeared to be the marker of Beijing genotype rather than being the cause of a long-term TB history.

\section{Discussion}

Drug-resistant tuberculosis is a serious threat to public health worldwide. The varied genetic mutations in $M$. tuberculosis after its onset depend on the microenvironment in which the tuberculosis bacilli exist. The selection process resulting from the antibiotic therapy may be one of the main reasons for the occurrence of SNPs. The occurrence of SNPs in the genomes from the patients in whom the drug susceptibility changed from sensitive to resistant strains is well known $[8,9]$. Recent studies involving re-sequencing of a large number of

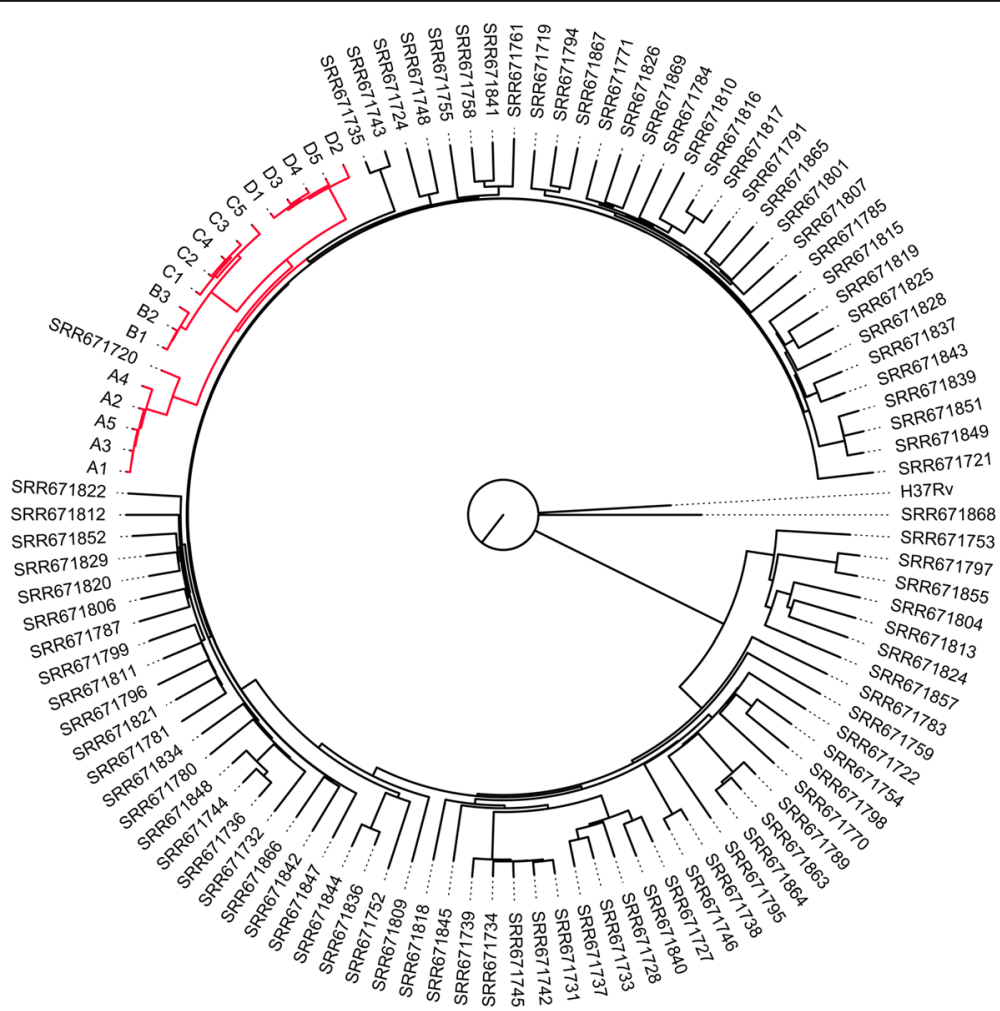

Fig. 5 Microevolution of the tested refractory TB population. Maximum likelihood phylogenetic trees of the M. tuberculosis drug-resistant isolates were created using the SNPs of the whole genome sequences by kSNP3 with a k-mer length of 19 . The red branch includes all of our sequenced refractory TB strains 
clinical isolates have also provided important insights into the complex evolution patterns of the TB pathogen $[3,4,36,37]$. This study further confirmed the genetic changes when the isolates in pre-XDR patients were treated with more complex regimens in an attempt to explain the prolonged histories of some tuberculosis patients (Table 2).

The genomic changes of the isolates within the individuals were significantly different. Only a few mutations, were found between baseline and re-infection, and there were no different sites between the baseline and relapse strains in $82 \%$ relapse cases [10]. In longitudinal isolates from 30 individuals and 25 families, the estimated rate of change in DNA sequences was 0.5 SNPs, per genome, per year (95\% Confidence interval 0.3-0.7), with the divergence rarely higher than five SNPs in 3 years [11]. However, Ford et al. suggested that the rate of change in DNA sequences is a constant of 0.5 SNP per genome per year in latent, active, or re-activated disease over the same period of time [38]. In this study, the mean mutation rate was 3.2 SNP per genome per year (Fig. 3c). This indicates that the complex treatments might increase the genetic changes including the compensatory substitutions and the non-coding regions (Fig. 3b). Furthermore, based on the previous reports, we found that the SNPs of each isolate ranged from 1300 to 1500 bases [10, 39], while the SNPs among the strains with Beijing genotypes range from 200 to 500 bases $[5,19]$. The SNPs of the intra-isolates were about 22.4 bases at the early sensitive stage [10].

Transient mutations were reported early in a case that evolved from a susceptible ancestor to XDR-TB [9]. The genetic loci, in this case, were not stable and changed their priority of bases under the antibiotic circumstance [9], implying the microheterogeneity in the cultured sputum population. In this study, many loci with microheterogeneity were found in the genome of the isolates at each time point and were repeatedly found in the isolates from different patients. The transient mutations were observed higher in the nonsynonymous (63.6\%) and non-coding regions $(22.7 \%)$ in intra-patient than that compared with H37Rv, with $54.6 \%$ nonsynonymous and $15.0 \%$ non-coding SNPs (Fig. 3b). The transient mutations suggest that the unstable genetic sites exist in the $M$. tuberculosis genome with the probability of a strong positive selection under the pressure of antibiotics.

The compensatory substitutions are common in drug-resistant TB that reflects to some extent the transmissibility and prevalence of drug-resistant tuberculosis in a population. With an increase in the number of sequenced isolates, more and more compensatory mutation types were observed in M. tuberculosis [3, 13, $40,41]$. For rifampicin-resistant isolates, the occurrence of compensatory substitutions in RpoA and RpoC were frequently found in isolates carrying the rpoB S450 L mutation (equivalent to Escherichia coli S531 L) [3, 13, $40,42]$. In this study, compensatory substitutions were only found in $\mathrm{RpoC}$ (I491T) in the isolates from patient D.

A large number of mutations related to the drug resistance have been found worldwide. In this study, the isolates from four pre-XDR patients had mutations in genes like katG and inhA promoter for INH, rpoB for RIF, rrs and eis for Amk/CPM, and gyrA for LFX [5, 37]. Some other mutations related to the resistance of EMB (embB, Rv3806c, proZ, pyrG), PZA (pncA), and PAS $(\mathrm{fol} C)$ were also found in some of the isolates, with the genotype in keeping with the phenotype (Table 2). In addition, some genes related to the multidrug resistance were listed in Table 2, such as Rv3756c (proZ), Rv1699 (pyrG), Rv1129c, Rv0323c, Rv0404 (fadD30), Rv0565c and Rv2080 (lppJ), which need to be further verified by molecular and biochemical methods.

In this study, we tried to find the characteristics of the SNPs and Indels specific to the four refractory pre-XDR TB patients, in comparison with the not refractory pre-XDR TB published data [5]. The phylogenetic tree demonstrated that the isolates in this study are phylogenetically close to each other (Fig. 5 ), indicating that the SNPs found in the refractory 18 tuberculosis isolates were potentially specific, of which 93 SNPs exhibited significantly different percentages (Additional file 4: Table S3). Although the main deletions in cut1 [43], plcD, and wag22 occurred in all of the M. tuberculosis populations, our data showed negative results, indicating that these deletions were specific for drug-resistant

Table 3 Comparison of the deleted genes that occurred in the Beijing genotype and the non-Beijing genotype isolates

\begin{tabular}{|c|c|c|c|c|c|}
\hline \multirow{2}{*}{$\begin{array}{l}\text { Gene } \\
\text { name }^{a}\end{array}$} & \multirow{2}{*}{$\begin{array}{l}\text { Gene } \\
\text { length } \\
\text { (bp) }\end{array}$} & \multicolumn{2}{|c|}{ Beijing genotype (n, \%) } & \multicolumn{2}{|c|}{ Non- Beijing Genotype (n, \%) } \\
\hline & & Resistant $(n=91, \%)$ & Sensitive $(n=21)$ & Resistant $(n=26)$ & Sensitive $(n=23)$ \\
\hline cut1 & 525 & 91,100 & $20,95.24$ & $13,50.00$ & $14,60.87$ \\
\hline plcD & 843 & $90,98.91$ & $20,95.24$ & $4,15.38$ & $7,30.43$ \\
\hline $\operatorname{wag} 22$ & 2745 & $78,85.71$ & 21,100 & $21,80.77$ & $16,69.56$ \\
\hline
\end{tabular}

\#The genome sequence of Mycobacterium tuberculosis H37Rv (NC_000962.3) was used as reference

${ }^{\mathrm{a}}$ Deletion occurred in all of the populations in all three genes 
isolates, and they might be characteristics of the Beijing lineage (Table 3).

\section{Conclusions}

In summary, four pre-XDR tuberculosis cases were enrolled in this study to test the whole genome changes of the 18 serial isolates from these patients during the elaborate treatment. The results indicate that each patient with a long history of TB is not infected by mixed strains (pathogen population). The SNPs found by whole genome sequencing showed transient and fixed mutations. Mutations related to the drug-resistance were fixed under the continuous drug pressure. We also found a transient mutation in some cases, suggesting that the treatment regimen could eradicate some bacterial population at different stages, except for the mutations related to the drug resistance. Of all the SNPs and Indels, 93 SNPs exhibited significantly different percentages in this study. These results indicate that the genetic changes might be partly responsible for refractory pre-XDR TB in the four selected TB cases.

\section{Additional files}

Additional file 1: Table S1. Sequencing statistics of the $18 \mathrm{M}$. tuberculosis isolates. Note: A, B, C, and D indicate the four cases, and the number following them indicates the different isolates. Sampling Time means the time that the samples collected from the four cases. H37Rv coverage (\%) means the percentage of the H37Rv genome covered by mapping reads. *The number of SNPs was assessed relative to H37Rv (NC_000962.3). (XLSX 11 kb)

Additional file 2: Figure S1. Boxplot graph showing the different types of SNP mutations. (PNG $117 \mathrm{~kb}$ )

Additional file 3: Table S2. Detailed information of SNPs within four patients. (XLSX $14 \mathrm{~kb}$ )

Additional file 4: Table S3. Ninety three SNPs exhibited significantly different percentages (adjusted $p$-value $<0.01$ ) between our sequenced genomes and the reference drug-resistant Beijing genomes. (XLSX $32 \mathrm{~kb}$ )

Additional file 5: Figure S2. Distribution of SNPs according to the Clusters of Orthologous Groups (COG) classification. (PNG 41 kb)

\section{Abbreviations}

Amk: Amikacin; COG: Clusters of Orthologous Groups; CPM: Capreomycin; DSTs: Drug susceptibility tests; EMB: Ethambutol; ETH: Ethionamide; INH: Isoniazid; LFX: Levofloxacin; MDR: Multidrug resistance; OFX: Ofloxacin; PAS: p-aminosalicylic acid sodium; PZA: Pyrazinamide; RIF: Rifampin; SM: Streptomycin; SNPs: Single nucleotide polymorphisms; TB: Tuberculosis; VNTR: Variable-number tandem repeat; XDR: Extensive drug resistance

\section{Acknowledgements}

The authors would also like to acknowledge the Beijing Bio-Bank of Clinical Resources-Tuberculosis, BBCR-Tuberculosis (D131100005313012) at Beijing Chest Hospital for providing all of the type isolates and clinical isolates used in this study.

\section{Funding}

The work was supported by the Key Project Specialized for Infectious Diseases of the Chinese Ministry of Health (2013ZX10003009), Tongzhou District Science and Technology Committee (KJ2017CX053), and the National Natural Science Foundation of China (NSFC) (31601081 and 81871691).

\section{Availability of data and materials}

The raw sequencing data of $M$. tuberculosis isolates reported in this study have been deposited in GenBank under accession numbers SRR3742653SRR3742670.

\section{Authors' contributions}

ZS and BZ conceived and designed the experiments. YX, SC, and ZS collected the clinical isolates and analyzed the clinical information and experimental data. YX, and JW conducted the drug-resistance tests. FL and $\mathrm{YH}$ performed the genome sequencing, and data analysis. FL, ZS, YX, JW, and SC wrote the manuscript. FL, YH, ZS, and BZ discussed the results and critiqued the manuscript. All authors read and approved the final manuscript.

\section{Ethics approval and consent to participate}

The ethical committee of Beijing Chest Hospital approved this study. The study methodologies conformed to the standards set by the Declaration of Helsinki and the experiments were undertaken with the understanding and written consent of each patient. All the authors ensured that all risks were minimized and the subjects were not injured.

\section{Consent for publication}

Not applicable.

\section{Competing interests}

All authors declare that they have no competing interests.

\section{Publisher's Note}

Springer Nature remains neutral with regard to jurisdictional claims in published maps and institutional affiliations.

\section{Author details}

${ }^{1}$ Institute of Chinese Materia medica, China Academy of Chinese Medical Science, Beijing 100700, China. ${ }^{2}$ National Tuberculosis Clinical Laboratory, Beijing Chest Hospital, Capital Medical University, Beijing 101149, China. ${ }^{3}$ CAS key Laboratory of Pathogenic Microbiology and Immunology, Institute of Microbiology, Chinese Academy of Science, Beijing 100101, China. ${ }^{4}$ Beijing Key Laboratory in Drug Resistant Tuberculosis Research, Beijing Tuberculosis \& Thoracic Tumor Research Institute, Beijing 101149, China.

Received: 13 November 2017 Accepted: 14 August 2018

Published online: 29 August 2018

\section{References}

1. WHO: Global Tuberculosis Report 2016 http://www.who.int/tb/publications/ global_report/en/. 2016.

2. Falzon D, Schunemann HJ, Harausz E, Gonzalez-Angulo L, Lienhardt C, Jaramillo E, Weyer K. World Health Organization treatment guidelines for drug-resistant tuberculosis, 2016 update. Eur Respir J. 2017;49

3. Casali N, Nikolayevskyy V, Balabanova Y, Harris SR, Ignatyeva O, Kontsevaya I, Corander J, Bryant J, Parkhill J, Nejentsev S, et al. Evolution and transmission of drug-resistant tuberculosis in a Russian population. Nat Genet. 2014;46: 279-86.

4. Walker TM, Kohl TA, Omar SV, Hedge J, Del Ojo Elias C, Bradley P, Iqbal Z, Feuerriegel S, Niehaus KE, Wilson DJ, et al. Whole-genome sequencing for prediction of Mycobacterium tuberculosis drug susceptibility and resistance: a retrospective cohort study. Lancet Infect Dis. 2015;15:1193-202.

5. Zhang H, Li D, Zhao L, Fleming J, Lin N, Wang T, Liu Z, Li C, Galwey N, Deng J, et al. Genome sequencing of 161 Mycobacterium tuberculosis isolates from China identifies genes and intergenic regions associated with drug resistance. Nat Genet. 2013;45:1255-60.

6. Lieberman TD, Wilson D, Misra R, Xiong LL, Moodley P, Cohen T, Kishony R. Genomic diversity in autopsy samples reveals within-host dissemination of HIV-associated Mycobacterium tuberculosis. Nat Med. 2016;22:1470-4.

7. Bloemberg GV, Keller PM, Stucki D, Trauner A, Borrell S, Latshang T, Coscolla M, Rothe T, Homke R, Ritter C, et al. Acquired resistance to Bedaquiline and Delamanid in therapy for tuberculosis. N Engl J Med. 2015;373:1986-8.

8. Sun G, Luo T, Yang C, Dong X, Li J, Zhu Y, Zheng H, Tian W, Wang S, Barry CE 3rd, et al. Dynamic population changes in Mycobacterium tuberculosis during acquisition and fixation of drug resistance in patients. J Infect Dis. 2012;206:1724-33. 
9. Eldholm V, Norheim G, von der Lippe B, Kinander W, Dahle UR, Caugant DA, Mannsaker T, Mengshoel AT, Dyrhol-Riise AM, Balloux F. Evolution of extensively drug-resistant Mycobacterium tuberculosis from a susceptible ancestor in a single patient. Genome Biol. 2014;15:490.

10. Bryant JM, Harris SR, Parkhill J, Dawson R, Diacon AH, van Helden P, Pym A, Mahayiddin AA, Chuchottaworn C, Sanne IM, et al. Whole-genome sequencing to establish relapse or re-infection with Mycobacterium tuberculosis: a retrospective observational study. Lancet Respir Med. 2013;1: 786-92.

11. Walker TM, Ip CL, Harrell RH, Evans JT, Kapatai G, Dedicoat MJ, Eyre DW, Wilson DJ, Hawkey PM, Crook DW, et al. Whole-genome sequencing to delineate Mycobacterium tuberculosis outbreaks: a retrospective observational study. Lancet Infect Dis. 2013;13:137-46.

12. Li QJ, Jiao WW, Yin QQ, Xu F, Li JQ, Sun L, Xiao J, Li YJ, Mokrousov I, Huang $\mathrm{HR}$, Shen AD. Compensatory mutations of rifampin resistance are associated with transmission of multidrug-resistant Mycobacterium tuberculosis Beijing genotype strains in China. Antimicrob Agents Chemother. 2016;60:2807-12.

13. Casali N, Nikolayevskyy V, Balabanova Y, Ignatyeva O, Kontsevaya I, Harris SR, Bentley SD, Parkhill J, Nejentsev S, Hoffner SE, et al. Microevolution of extensively drug-resistant tuberculosis in Russia. Genome Res. 2012;22: 735-45.

14. Reeves AZ, Campbell PJ, Willby MJ, Posey JE. Disparities in capreomycin resistance levels associated with the rrs A1401G mutation in clinical isolates of Mycobacterium tuberculosis. Antimicrob Agents Chemother. 2015; 59:444-9.

15. Perez-Lago L, Comas I, Navarro Y, Gonzalez-Candelas F, Herranz M, Bouza E, Garcia-de-Viedma D. Whole genome sequencing analysis of intrapatient microevolution in Mycobacterium tuberculosis: potential impact on the inference of tuberculosis transmission. J Infect Dis. 2014;209:98-108.

16. McGrath M, Gey van Pittius NC, van Helden PD, Warren RM, Warner DF. Mutation rate and the emergence of drug resistance in Mycobacterium tuberculosis. J Antimicrob Chemother. 2014;69:292-302.

17. Trauner A, Liu Q, Via LE, Liu X, Ruan X, Liang L, Shi H, Chen Y, Wang Z, Liang $R$, et al. The within-host population dynamics of Mycobacterium tuberculosis vary with treatment efficacy. Genome Biol. 2017;18:71.

18. Sun Z, Chao Y, Zhang X, Zhang J, Li Y, Qiu Y, Liu Y, Nie L, Guo A, Li C. Characterization of extensively drug-resistant Mycobacterium tuberculosis clinical isolates in China. J Clin Microbiol. 2008;46:4075-7.

19. Kamerbeek J, Schouls L, Kolk A, van Agterveld M, van Soolingen D, Kuijper S, Bunschoten A, Molhuizen H, Shaw R, Goyal M, van Embden J. Simultaneous detection and strain differentiation of Mycobacterium tuberculosis for diagnosis and epidemiology. J Clin Microbiol. 1997;35: 907-14.

20. Sun Z, Li W, Xu S, Huang H. The discovery, function and development of the variable number tandem repeats in different Mycobacterium species. Crit Rev Microbiol. 2016;42:738-58.

21. Cox MP, Peterson DA, Biggs PJ. SolexaQA: at-a-glance quality assessment of Illumina second-generation sequencing data. BMC Bioinform. 2010;11:485.

22. Luo R, Liu B, Xie Y, Li Z, Huang W, Yuan J, He G, Chen Y, Pan Q, Liu Y, et al. SOAPdenovo2: an empirically improved memory-efficient short-read de novo assembler. Gigascience. 2012;1:18.

23. O'Toole RF, Gautam SS. Limitations of the Mycobacterium tuberculosis reference genome H37Rv in the detection of virulence-related loci. Genomics. 2017;109:471-4.

24. Li R, Yu C, Li Y, Lam TW, Yiu SM, Kristiansen K, Wang J. SOAP2: an improved ultrafast tool for short read alignment. Bioinformatics. 2009;25:1966-7.

25. Li R, Li Y, Fang X, Yang H, Wang J, Kristiansen K, Wang J. SNP detection for massively parallel whole-genome resequencing. Genome Res. 2009;19: 1124-32.

26. Darling AC, Mau B, Blattner FR, Perna NT. Mauve: multiple alignment of conserved genomic sequence with rearrangements. Genome Res. 2004;14: 1394-403.

27. Fisher RA. On the interpretation of $\times 2$ from contingency tables, and the calculation of P. J R Stat Soc. 1922;85:87-94.

28. Gardner SN, Slezak T, Hall BG. KSNP3.0: SNP detection and phylogenetic analysis of genomes without genome alignment or reference genome. Bioinformatics. 2015;31:2877-8.

29. Bandelt HJ, Forster P, Rohl A. Median-joining networks for inferring intraspecific phylogenies. Mol Biol Evol. 1999;16:37-48.
30. Caminero JA, Sotgiu G, Zumla A, Migliori GB. Best drug treatment for multidrug-resistant and extensively drug-resistant tuberculosis. Lancet Infect Dis. 2010;10:621-9.

31. WHO. Companion Handbook to the WHO Guidelines for the Programmatic Management of Drug-Resistant Tuberculosis. In: Companion Handbook to the WHO Guidelines for the Programmatic Management of Drug-Resistant Tuberculosis: Geneva, WHO Guidelines Approved by the Guidelines Review Committee; 2014

32. Cronan JE Jr, Waldrop GL. Multi-subunit acetyl-CoA carboxylases. Prog Lipid Res. 2002:41:407-35.

33. Kim KH, An DR, Song J, Yoon JY, Kim HS, Yoon HJ, Im HN, Kim J, Kim DJ, Lee SJ, et al. Mycobacterium tuberculosis Eis protein initiates suppression of host immune responses by acetylation of DUSP16/MKP-7. Proc Natl Acad Sci U S A. 2012;109:7729-34.

34. Wei J, Dahl JL, Moulder JW, Roberts EA, O'Gaora P, Young DB, Friedman RL. Identification of a Mycobacterium tuberculosis gene that enhances mycobacterial survival in macrophages. J Bacteriol. 2000;182:377-84.

35. Calvanese L, Falcigno L, Maglione C, Marasco D, Ruggiero A, Squeglia F, Berisio R, D'Auria G. Structural and binding properties of the PASTA domain of PonA2, a key penicillin binding protein from Mycobacterium tuberculosis. Biopolymers. 2014;101:712-9.

36. Merker M, Blin C, Mona S, Duforet-Frebourg N, Lecher S, Willery E, Blum MG Rusch-Gerdes S, Mokrousov I, Aleksic E, et al. Evolutionary history and global spread of the Mycobacterium tuberculosis Beijing lineage. Nat Genet. 2015; 47:242-9.

37. Manson AL, Cohen KA, Abeel T, Desjardins CA, Armstrong DT, Barry CE 3rd, Brand J, Consortium TBGG, Chapman SB, Cho SN, et al. Genomic analysis of globally diverse Mycobacterium tuberculosis strains provides insights into the emergence and spread of multidrug resistance. Nat Genet. 2017;49:395-402.

38. Ford CB, Lin PL, Chase MR, Shah RR, lartchouk O, Galagan J, Mohaideen N, loerger TR, Sacchettini JC, Lipsitch M, et al. Use of whole genome sequencing to estimate the mutation rate of Mycobacterium tuberculosis during latent infection. Nat Genet. 2011;43:482-6.

39. Liu F, Hu Y, Wang Q, Li HM, Gao GF, Liu CH, Zhu B. Comparative genomic analysis of Mycobacterium tuberculosis clinical isolates. BMC Genomics. 2014;15:469

40. Comas I, Borrell S, Roetzer A, Rose G, Malla B, Kato-Maeda M, Galagan J, Niemann S, Gagneux S. Whole-genome sequencing of rifampicin-resistant Mycobacterium tuberculosis strains identifies compensatory mutations in RNA polymerase genes. Nat Genet. 2011;44:106-10.

41. Sherman DR, Mdluli K, Hickey MJ, Arain TM, Morris SL, Barry CE 3rd, Stover CK. Compensatory ahpC gene expression in isoniazid-resistant Mycobacterium tuberculosis. Science. 1996;272:1641-3.

42. Cohen KA, Abeel T, Manson McGuire A, Desjardins CA, Munsamy V, Shea TP, Walker BJ, Bantubani N, Almeida DV, Alvarado L, et al. Evolution of extensively drug-resistant tuberculosis over four decades: whole genome sequencing and dating analysis of Mycobacterium tuberculosis isolates from KwaZulu-Natal. PLoS Med. 2015:12:e1001880.

43. Parker SK, Curtin KM, Vasil ML. Purification and characterization of mycobacterial phospholipase a: an activity associated with mycobacterial cutinase. J Bacteriol. 2007;189:4153-60.
Ready to submit your research? Choose BMC and benefit from:
- fast, convenient online submission
- thorough peer review by experienced researchers in your field
- rapid publication on acceptance
- support for research data, including large and complex data types
- gold Open Access which fosters wider collaboration and increased citations
- maximum visibility for your research: over $100 \mathrm{M}$ website views per year
At BMC, research is always in progress. 